\title{
Delta Neutrophil Index as a Prognostic Marker in the Pediatric Intensive Care Unit
}

\author{
In Suk Sol, M.D., Hyun Bin Park, M.D., Min Jung Kim, M.D., Seo Hee Yoon, M.D., Ph.D., Yoon Hee Kim, M.D., \\ Kyung Won Kim, M.D., Ph.D, Myung Hyun Sohn, M.D., Ph.D, and Kyu-Earn Kim, M.D., Ph.D
}

Department of Pediatrics, Severance Hospital, Institute of Allergy, Brain Korea 21 PLUS Project for Medical Science, Yonsei University College of Medicine, Seoul, Korea

Background: The delta neutrophil index (DNI) is a useful marker for diagnosing and predicting the prognosis of sepsis. The purpose of this study was to investigate the usefulness of DNI as a prognostic marker in patients within the pediatric intensive care unit (PICU), as well as its association with other prognostic factors.

Methods: A total of 516 children admitted to Severance Children's Hospital PICU from December 2009 to February 2015 were analyzed. DNI was measured on the day of PICU admission. Mortality was defined as death within 28 days following PICU admission. Results: The median value of DNI was $1.2 \%$ (interquartile range [IQR] 0-4.3\%) in the survivor group and 9.5\% (IQR 2.3-20.8\%) in the non-survivor group, and the difference was statistically significant $(p<0.001)$. DNI was significantly positively correlated with ICU scores such as Pediatric Index of Mortality 3 and Pediatric Risk of Mortality III, as well as with C-reactive protein and lactate levels. The area under the receiver operating characteristic curve of DNI for mortality was 0.748 ( $95 \% \mathrm{Cl}: 0.687-0.808)$ and the cut-off value was $4.95 \%$.

Conclusions: The initial DNI level can be considered a useful indicator for predicting prognosis in PICU patients.

Key Words: child; intensive care unit; mortality; prognostic factor.

\section{Introduction}

The outcome of critically ill children recovering from life-threatening diseases in intensive care situations has improved owing to advancing diagnostic and therapeutic methods.[1] Clinicians recognized the importance of identifying patients with the highest risk of mortality among those admitted to the pediatric intensive care unit (PICU), and of proper monitoring and appropriate intervention and treatment. They also recognized the need to observe and treat highrisk patients more aggressively, using appropriate indicators to predict mortality.

Many studies of mortality predictors in PICU patients have been performed, and ICU scoring systems such as the pe-

Received on March 14, 2016 Revised on May 18, 2016 Accepted on May 22, 2016

Correspondence to: Myung Hyun Sohn, Department of Pediatrics, Institute of Allergy, Severance Children's Hospital, Yonsei University College of Medicine, 50 Yonsei-ro, Seodaemun-gu, Seoul 03722, Korea

Tel: +82-2-2228-2062, Fax: +82-2-393-9118 E-mail: MHSOHN@yuhs.ac

*No potential conflict of interest relevant to this article was reported. diatric risk of mortality III (PRISM-III), pediatric index of mortality 2 (PIM2), PIM3, and pediatric logistic organ dysfunction have been validated and are being used in clinical practice.[2-5] In addition, studies on the association between mortality and laboratory biomarkers such as C-reactive protein (CRP), platelet count, procalcitonin, transthyretin and eosinophil count in adult ICU patients 
have also been performed.[6-11]

The delta neutrophil index (DNI) is a parameter measured by a specific type of automated blood cell analyzer, the ADVIA2120 Hematology System and reflects circulating immature granulocytes (IGs).[12] The ADVIA 2120 Hematology System is a flow cytometry-based hematologic analyzer comprised of two independent white blood cell (WBC) analysis methods (peroxidase and lobularity/ nuclear density channels).[13,14] The difference between the leukocyte differentials assayed in the peroxidase channel and those measured in the nuclear lobularity channel is defined as the DNI.[12]

In a previous study in adult patients with suspected sepsis, the DNI was helpful in evaluating clinical severity. [12] In another study, the DNI elevated in patients with sepsis or septic shock and overt disseminated intravascular coagulation (DIC) have been proposed as a marker of disease severity in patients with sepsis.[15] Meanwhile, DNI was suggested as a marker to discriminate adult onset Still's disease from sepsis.[16] IGs are also an indicator of a left shift occurring due to infection, and are a meaningful discriminator the presence of sepsis. It was also reported to be a predictor of infection and positive blood cultures.[17,18] In a study of infants, an increase in IG was associated with positive blood cultures.[19]

There have been some studies on the associations between DNI/IGs and the severity of sepsis, but no research has been conducted on the relationship between these parameters and survival in critical ill children. Therefore, the purpose of this study was to investigate the usefulness of DNI in pediatric patients admitted to the PICU as a predictor of survival and prognosis, in addition to its association with other known prognostic factors.

\section{Materials and Methods}

\section{1) Patients and variables}

Children under the age of 18 who were admitted to the PICU at Severance Children's Hospital, Seoul, Korea between December 2009 and February 2015 were included in this study. Patients originated from the emergency room or the general ward. The medical records of 516 children were analyzed retrospectively. Patients who died or were transferred to the general ward within $24 \mathrm{~h}$ after ICU admission were excluded. The collected data included patient age, sex, ICU admission diagnosis, absence or presence of mechanical ventilation within $24 \mathrm{~h}$ after ICU admission, length of ICU stay, blood and sputum culture results, in addition to complete blood cell (CBC) count, coagulation parameters, erythrocyte sedimentation rate (ESR), and CRP at admission. Additionally, PRISM III,[20] PIM2,[21] and PIM3[22] were calculated to define the severity of a patient's condition within the first $24 \mathrm{~h}$ of ICU admission. Survival or death was defined as the 28-day fatality following ICU admission. DNI values were recorded on the day of ICU admission. Patients were divided into the following three groups based on the initial DNI level: no increase in DNI $(<5 \%)$, low DNI (5-20\%), and intermediate-to-high DNI (>20\%). [12] This study was approved by the Institutional Review Board of Severance Hospital (protocol no. 4-2015-1212).

\section{2) DNI measurement}

Blood samples for DNI measurement were obtained by venipuncture or indwelling catheter. They were drawn into ethylene diamine tetra acetic acid-treated tubes and analyzed within $24 \mathrm{~h}$ of blood sampling. DNI was measured using an ADVIA2120 Hematology System (Siemens Healthcare Diagnostics, Forchheim, Germany, 2010).[13,14] After lysis of red blood cells (RBC), a tungsten-halogen-based optical system of the peroxidase channel measures the forward light scatter (cell size) and absorbance (stain intensity) to count and distinguish lymphocytes, monocytes, neutrophils, and eosinophils based on size and peroxidase content. The laser diode-based optical system of the lobularity/nuclear density channel uses an acidic reagent to lyse RBCs and platelets and strips the cytoplasmic membrane from all of the WBCs except basophils. Cells are counted and classified according to size (basophils are recognized as the largest cells because they have a cytoplasmic membrane), lobularity 
and nuclear density. This channel provides valuable information about the degree of maturity of each WBC's nucleus by measuring its lobularity and density, in addition to reporting the WBC count and basophil count. The DNI can therefore be calculated using the following formula: $\mathrm{DNI}=$ (the neutrophil subfraction and the eosinophil subfraction measured in the myeloperoxidase [MPO] channel by the cytochemical MPO reaction) - (the polymorphonuclear neutrophil [PMN] subfraction measured in the nuclear lobularity channel by the reflected light beam).[15]

\section{3) Statistical analysis}

Categorical variables are represented as numeric values (percentages) and continuous variables as median values (interquartile ranges [IQRs]). Groups were compared by the chi-square test or Fisher's exact test for categorical variables, and the Mann-Whitney $U$ test, as appropriate. The associations between DNI and the ICU scoring system values and other laboratory parameters were examined using Spearman's method.

Receiver operating characteristic (ROC) curves and the respective area under the curve (AUC) of DNI at ICU admission for predicting mortality were calculated.[23] The Youden[24] method was used to select an "optimal" DNI level for predicting mortality. Patients were stratified into two groups according to this optimal level for analyses. Kaplan-Meier curves were constructed using these strata and compared using log-rank testing. Statistical analyses were performed using SPSS 20 (SPSS, Inc., Chicago, IL, USA) and $\mathrm{R}$ software ( $\mathrm{R}$ version 3.2.4). P-values $<0.05$ were considered statistically significant for all analyses.

\section{Results}

\section{1) Characteristics of the study population}

A total of 516 children were analyzed in this study. Among them, 422 patients survived and 94 died (mortality rate of $18.2 \%$ ). The reasons for ICU admission included respiratory disease, such as pneumonia, bron- chiolitis obliterans or laryngomalacia, in 270 patients (52.3\%); neurological problem, such as epilepsy, hypoxic ischemic encepharopathy or encephalitis, in 91 patients (17.6\%); nephrologic problem, such as renal failure or hemolytic uremic syndrome, in 24 patients (4.7\%); postoperative care, in 39 patients (7.6\%); hemato-oncologic problem, such as tumor lysis syndrome or neutropenic fever in patients having chemotherapy, in 36 patients (7.0\%); gastrointestinal problem, such as gastrointestinal bleeding or hepatic failure, in 30 patients $(5.8 \%)$; metabolic disorder in 10 patients $(1.9 \%)$ and other problem in 30 patients $(5.8 \%)$.

Baseline characteristics of the study population, shown in Table 1, were compared between the survivor and nonsurvivor groups. The DNI was significantly higher in the non-survivor group (median [IQR] 9.5 [2.3-20.8]) than in the survivor group $(1.2[0-4.3])(\mathrm{p}<0.001)$. The platelet count was lower $(\mathrm{p}<0.001)$ and CRP was higher $(\mathrm{p}<$ 0.001 ) in the non-survivor group. Scores on three different ICU scoring systems (PRISM III, PIM2, and PIM3) that evaluate the severity of the patient's condition were all significantly higher in the non-survivor group. Prothrombin time $(\mathrm{PT})(\mathrm{p}<0.001)$, international normalized ratio $(p<0.001)$, and $D$-dimer levels $(p=0.001)$ were significantly higher in the non-survivor group. No significant differences were observed in sex, positive sputum culture rates, ESR, and or antithrombin III (ATIII) levels between the survival and mortality groups.

\section{2) DNI level in the immunocompromised and immuno- competent patients}

Among 516 patients, 72 children were the immunocompromised patients who were defined as having current chemotherapy treatment, immunosuppressive therapy, or having undergone hematopoietic stem cell or solid organ transplantation. The DNI was significantly higher in the immunocompromised patients (median [IQR] 4.7 (0.3$30.6)]$ than in the immunocompetent patients $(1.6[0-5.3])$ $(\mathrm{p}=0.001)$. In the immunocompromised patients, 32 patients $(44.4 \%)$ were classified as the non-survival group and the DNI was higher in the non-survivor group (me- 
dian [IQR] $15.3[3.3-28.2])$ than in the survivor group (1.9 $[0-6.8])(\mathrm{p}=0.001)$.

\section{3) Comparisons of patients according to DNI level at ICU admission and the relationship between DNI and other parameters}

Table 2 compares characteristics among the three patient subgroups based on DNI level at ICU admission:
DNI $<5 \%(\mathrm{n}=366)$, DNI 5-20\% $(\mathrm{n}=111)$, and DNI $>$ $20 \%(\mathrm{n}=39)$. As DNI increased, the proportion of survivors declined $(p<0.001)$. The three ICU scores also increased parallel with increasing DNI $(p=0.001)$. Platelet levels were lowest in the high DNI group $(\mathrm{p}=0.005)$. DNI was significantly positively correlated with the three ICU scores ( $r=0.377, p<0.001$ for PRISM III; $r=0.274$, $\mathrm{p}<0.001$ for PIM2; and $\mathrm{r}=0.317, \mathrm{p}<0.001$ for PIM3)

Table 1. Characteristics of the study population

\begin{tabular}{|c|c|c|c|}
\hline & Survivor $(n=422)$ & Non-survivor $(n=94)$ & $\mathrm{p}$-value \\
\hline \multicolumn{4}{|l|}{ Clinical characteristics } \\
\hline Age (years) & $2.5(0.9-7.1)$ & $4.6(1.6-10.2)$ & 0.006 \\
\hline Male sex & $248(58.8)$ & $60(63.8)$ & 0.416 \\
\hline Positive blood culture & $18(4.3)$ & $11(12.5)$ & 0.009 \\
\hline Positive sputum culture & $64(16.7)$ & $10(12.5)$ & 0.405 \\
\hline Mechanical ventilation (1 h) & 377 (89.3) & $90(96.8)$ & 0.028 \\
\hline Mechanical ventilation (24 h) & $298(70.6)$ & $81(87.1)$ & 0.001 \\
\hline Length of ICU stay (days) & $9.5(5-20)$ & $6.0(2-15)$ & 0.001 \\
\hline \multicolumn{4}{|l|}{ ICU scoring systems } \\
\hline PRISM III & $5(2-10)$ & $19(10-28)$ & $<0.001$ \\
\hline PIM2 (\%) & $9.6(5.1-26.9)$ & 45.7 (22.2-81.3) & $<0.001$ \\
\hline PIM3 (\%) & $6.7(3.7-15.4)$ & $32.4(16.8-82.5)$ & $<0.001$ \\
\hline \multicolumn{4}{|l|}{ Hematologic variables } \\
\hline DNI (\%) & $1.2(0-4.3)$ & $9.5(2.3-20.8)$ & $<0.001$ \\
\hline WBC $\left(10^{3} / \mu \mathrm{L}\right)$ & 11160 (6913-17083) & 8355 (3348-17968) & 0.001 \\
\hline $\mathrm{Hb}(\mathrm{g} / \mathrm{dL})$ & $10.5 \pm 1.9$ & $10.1 \pm 2.8$ & 0.140 \\
\hline ANC $(/ \mu \mathrm{L})$ & 7730 (4140-12735) & 5415 (2119-12633) & 0.019 \\
\hline Platelets $\left(10^{3} / \mu \mathrm{L}\right)$ & 295.5 (178.5-428.3) & $136.0(60.0-266.3)$ & $<0.001$ \\
\hline $\mathrm{ESR}(\mathrm{mm} / \mathrm{hr})$ & $12.0(2-31)$ & $4.0(2-31)$ & 0.173 \\
\hline $\mathrm{CRP}(\mathrm{mg} / \mathrm{L})$ & $8.0(1.8-38.0)$ & $25.0(8.9-118.9)$ & $<0.001$ \\
\hline \multicolumn{4}{|l|}{ Coagulation parameters } \\
\hline PT (sec) & $12.5(11.4-14.0)$ & $15.2(12.2-20.8)$ & $<0.001$ \\
\hline INR & $1.1(1.0-1.2)$ & $1.4(1.1-1.8)$ & $<0.001$ \\
\hline aPTा (sec) & $32.4(28.3-38.0)$ & $45.1(30.4-79.5)$ & $<0.001$ \\
\hline Fibrinogen (mg/dL) & $258(181-347)$ & 218 (134-320) & 0.096 \\
\hline D-dimer (ng/mL) & $636(305-1328)$ & 1111 (453-3663) & 0.001 \\
\hline ATIII (\%) & $88(67-106)$ & $72(56-104)$ & 0.109 \\
\hline Lactate & $1.4(0.9-2.4)$ & $5.2(1.9-14.3)$ & $<0.001$ \\
\hline
\end{tabular}

Data are presented as the median (interquartile range) or number of patients (percentage).

p-values are from the chi-square test or Mann-Whitney $U$ test used to compare differences between the survivor and non-survivor groups.

ICU: intensive care unit; PRISM: pediatric risk of mortality; PIM: pediatric index of mortality; DNI: delta neutrophil index; WBC: white blood cells; Hb: hemoglobin; ANC: absolute neutrophil count; ESR: erythrocyte sedimentation rate; CRP: C-reactive protein; PT: prothrombin time; INR: international normalized ratio; aPT: activated partial thromboplastin time; ATII: antithrombin III. 
as well as lactate $(\mathrm{r}=0.264, \mathrm{p}<0.001)$ and $\mathrm{CRP}(\mathrm{r}=$ $0.344, \mathrm{p}<0.001)$.

\section{4) ROC curve of DNI and comparison of survival curves according to DNI cut-off level}

Figure 1 shows the ROC curve of DNI for predicting mortality. The AUC was 0.748 (95\% confidence interval $0.687-0.808 ; \mathrm{p}=0.001)$ and the optimal cut-off value was $4.95 \%$. Sensitivity and specificity were $63.8 \%$ and $78.7 \%$, respectively. With a cut-off value of $5.0 \%$, sensitivity and specificity were $63.4 \%$ and $78.7 \%$, respectively. The patients were divided into two groups according to their initial DNI levels; DNI $\geq 5.0 \%(\mathrm{n}=150)$ and $<$ $5.0 \%(\mathrm{n}=366)$. Kaplan-Meier curves showed the probability of survival in each group (Fig. 2). Patients with lower DNI levels had significantly longer survival time in the log-rank test $(\mathrm{p}<0.001)$.।

\section{Discussion}

We retrospectively analyzed the medical records of 516 children admitted to the PICU during a period of 5 years. At ICU admission, the median DNI was significantly

Table 2. Comparisons of study patients according to DNI level at ICU admission

\begin{tabular}{|c|c|c|c|c|}
\hline & $\begin{array}{l}\text { No increase in DNI }(<5) \\
(n=366)\end{array}$ & $\begin{array}{l}\text { Low DNI }(5-20) \\
\quad(n=111)\end{array}$ & $\begin{array}{l}\text { Intermediate-high DNI ( > 20) } \\
\qquad(\mathrm{n}=39)\end{array}$ & $\mathrm{p}$-value \\
\hline \multicolumn{5}{|l|}{ Clinical characteristics } \\
\hline Age (years) & $2.39(0.98-6.61)$ & $3.43(1.38-7.9)$ & $7.48(3.02-10.56)$ & 0.001 \\
\hline Male sex & $212(57.9)$ & $68(61.3)$ & $28(71.8)$ & 0.608 \\
\hline Survivors & $317(86.6)$ & $67(60.4)$ & $27(69.2)$ & $<0.001$ \\
\hline Mechanical ventilation & $246(67.2)$ & $99(90.0)$ & $34(87.2)$ & $<0.001$ \\
\hline \multicolumn{5}{|l|}{ ICU scoring systems } \\
\hline PRISM III & $5(1.0-10.0)$ & $10.0(6.0-18.0)$ & $20.0(10.0-31.0)$ & $<0.001$ \\
\hline PIM2 & $10.2(5.1-27.6)$ & $21.2(7.5-45.1)$ & $48.1(8.7-91.7)$ & $<0.001$ \\
\hline PIM3 & $7.1(3.6-15.9)$ & $12.8(5.4-29.3)$ & $43.8(6.8-93.2)$ & $<0.001$ \\
\hline \multicolumn{5}{|l|}{ Hematologic variables } \\
\hline WBC $\left(10^{3} / \mu \mathrm{L}\right)$ & 10.78 (6.58-15.68) & 13.29 (6.84-23.86) & $6.68(2.46-14.9)$ & 0.003 \\
\hline ANC $(/ \mu \mathrm{L})$ & $7,441(3,890-11,880)$ & $9,740(4,980-19,340)$ & $4,170(1,294-9,804)$ & 0.005 \\
\hline $\mathrm{Hb}(\mathrm{g} / \mathrm{dL})$ & $10.4(9.3-11.6)$ & $10.6(8.9-12.0)$ & $9.9(7.8-11.3)$ & 0.213 \\
\hline Platelets $\left(10^{3} / \mu \mathrm{L}\right)$ & $295(181-437)$ & $223(92-380)$ & $132(55-251)$ & $<0.001$ \\
\hline $\mathrm{ESR}(\mathrm{mm} / \mathrm{h})$ & $12.0(2.0-31.0)$ & $9.5(2.0-34.2)$ & $5.5(2.0-35.8)$ & 0.668 \\
\hline $\mathrm{CRP}(\mathrm{mg} / \mathrm{L})$ & $7.4(1.7-31.2)$ & $19.2(4.9-71.3)$ & $98.5(16.1-167.6)$ & $<0.001$ \\
\hline \multicolumn{5}{|l|}{ Coagulation parameters } \\
\hline PT (sec) & $12.5(11.4-14.0)$ & $13.0(11.6-16.2)$ & $16.3(13.5-22.6)$ & $<0.001$ \\
\hline INR & $1.10(1.00-1.21)$ & $1.13(1.02-1.42)$ & $1.45(1.23-1.98)$ & $<0.001$ \\
\hline $\mathrm{aPTT}(\mathrm{sec})$ & 31.7 (28.1-37.9) & 35.7 (29.7-52.3) & $45.8(32.7-61.4)$ & $<0.001$ \\
\hline D-dimer (ng/mL) & $539.0(284-1194)$ & 946.5 (543-2718) & $1444.5(449-7603)$ & $<0.001$ \\
\hline ATIII $(\% 0$ & $88.5(67.0-107.0)$ & $79.0(60.5-104.8)$ & $69.0(58.3-82.0)$ & 0.013 \\
\hline Lactate (mmol/L) & $1.4(0.9-2.4)$ & $2.1(1.2-4.9)$ & $4.6(1.6-14.0)$ & $<0.001$ \\
\hline
\end{tabular}

Data are presented as the median (interquartile range) or number of patients (percentage).

p-values are from the chi-square test or Kruskal-Wallis test used to compare differences between DNI groups.

ICU: intensive care unit, PRISM: pediatric risk of mortality, PIM: pediatric index of mortality, DNI: delta neutrophil, WBC: white blood cells, ANC: absolute neutrophil count, ESR: erythrocyte sedimentation rate, CRP: C-reactive protein, PT: prothrombin time, INR: international normalized ratio, aPTT: activated partial thromboplastin time, ATIII: antithrombin III. 


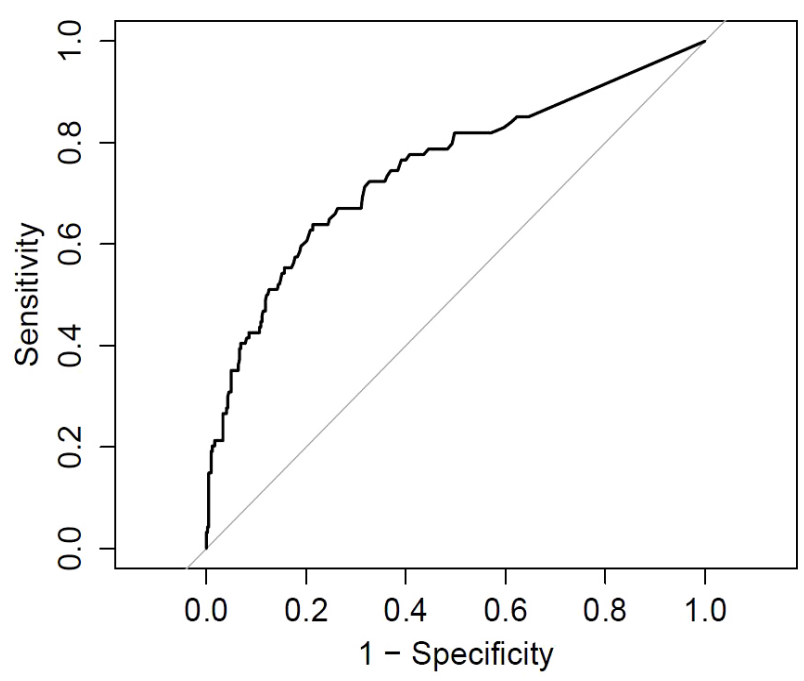

Fig. 1. Receiver operating characteristic curve of delta neutrophil index for survival prediction (area under the curve: 0.748; 95\% confidence interval: 0.687-0.808).

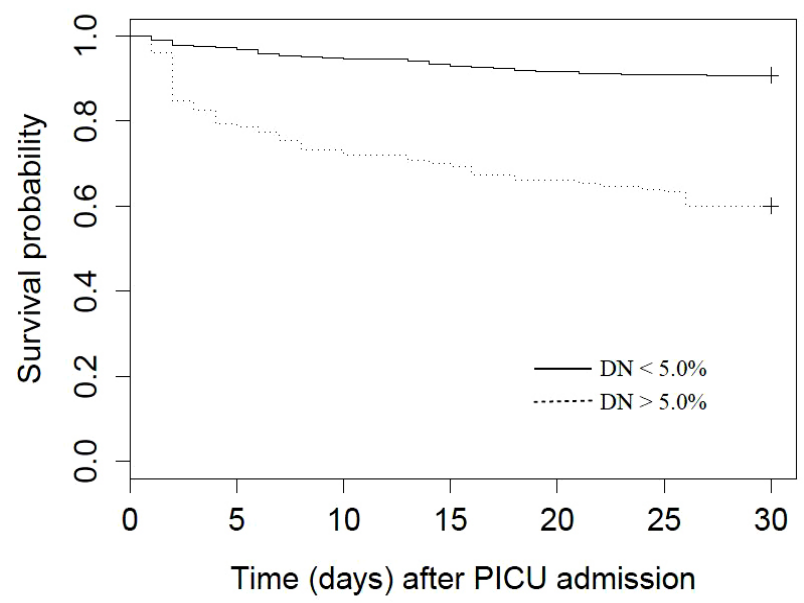

Fig. 2. Survival curves for patients with delta neutrophil index (DNI) $<5.0 \%$ (solid line) and DNI $\geq 5.0 \%$ (dotted line). DN: delta neutrophil; PICU: pediatric intensive care unit.

higher in the non-survivor group than in the survivor group. As admission DNI level increased, ICU scoring system values also increased. Additionally, the proportion of survivors decreased. The DNI at PICU admission was also positively correlated with ICU scores, as well as lactate and CRP and negatively correlated with platelet counts. The optimal cut-off value obtained by ROC curve analysis for predicting survival was $4.95 \%$, and mortality was significantly higher in patients with $\mathrm{DNI} \geq 5.0 \%$ than in patients with DNI $<5.0 \%$.
It is very challenging to identify patients with increased mortality risk early among critically ill children admitted to the PICU. The ideal marker of outcome in the PICU should be highly sensitive, specific, easily measurable, fast, and inexpensive and should correlate well with disease severity and prognosis. The DNI, which corresponds to the IG fraction in circulating blood, can be measured using an ADVIA-series automated cell analyzer. The DNI is an easily measurable parameter that is reported with the $\mathrm{CBC}$ count and a WBC differential. Thus, it has the advantage of no additional cost and being readily available. This study was performed to establish the clinical usefulness of the DNI by analyzing whether DNI predicts mortality and correlates with ICU scoring systems that reflect disease severity and other laboratory parameters in PICU patients.

In a previous study conducted in adult subjects by Nahm et al.[12] DNI was helpful in evaluating the clinical severity of patients with suspected sepsis and was significantly correlated with laboratory values of DIC; platelet count, PT, and ATIII level. In another study by Park et al.[15] an increased DNI value at ICU admission was significantly associated with the presence of sepsis or septic shock and overt DIC and was positively correlated with DIC score but not WBC count and absolute neutrophil count in adult medical ICU patients clinically suspected of sepsis at the time of ICU admission. Consequently, the authors suggested that an elevated DNI might reflect the disease severity of patients with sepsis and could be related to a hypercoagulable state associated with PT, activated partial thromboplastin time (aPTT), ATIII level, and platelet count. When analyzing all 516 participants, the DNI was also positively correlated with PT, aPTT, D-dimer level and platelet count. However, the ATIII level was not significantly different between survivors and non-survivors, although it was weakly correlated with DNI $(\mathrm{r}=-0.166, \mathrm{p}<0.015)$. This discrepancy may be due to differences in the study subjects, as previous studies considered only adult patients with clinically suspected or diagnosed sepsis, whereas this study included a heterogeneous sample of pediatric 
patients and focused on mortality prediction.

In this study, the optimal cut-off value of DNI to predict mortality was $4.95 \%$. In comparison, previous studies on circulating IG level, which is reflected by DNI, proposed a cut-off value of 3\% for determining the presence of sepsis.[17,18] In a report by Park et al.[15] it was suggested that the optimal cut-off value of DNI was $6.5 \%$ to predict sepsis or septic shock, and if the DNI increased by more than $4-6 \%$, careful attention was required to detect possible concomitant DIC and/or sepsis or septic shock in patients with suspected sepsis. One reason that accounts for the difference between the cut-off value in previous studies and ours is that the presence of severe sepsis/septic shock was the primary outcome in previous studies, whereas survival status was our primary outcome.

There are some limitations in the current study. First, this study was a retrospective review of medical records. Large-scale prospective studies will be helpful in elucidating the role of DNI in reflecting disease severity and predicting mortality. Second, because this study was conducted in a single medical PICU, our conclusions are limited to medical PICU patients and cannot be applied to all ICU patients including surgical problem. Lastly, Further study should include that usefulness and meaning of DNI in subgroup not belong to sepsis or infection. However, this study is the first study to demonstrate that the DNI can be used as a prognostic marker in a heterogeneous population of critically ill pediatric patients and even in the immunocompromised patients in PICU, unlike previous studies that considered only patients with suspected or diagnosed sepsis. Therefore, we suggest that DNI be considered a new indicator for predicting mortality in PICU patients.

In conclusion, DNI is associated with disease severity and mortality rate, and has the potential to serve as a prognostic marker in PICU patients. Accordingly, determining the DNI level at ICU admission can be helpful in deciding whether additional and more invasive diagnostic procedures are required or whether therapeutic interventions should be modified. Measurement of the DNI in PICU patients may add significant information to that provided by markers and scoring systems already in use for assessing disease severity and predicting mortality.

\section{ORCID}

In Suk Sol http://orcid.org/0000-0003-2470-9682

Min Jung Kim http://orcid.org/0000-0002-5634-9709

Seo Hee Yoon http://orcid.org/0000-0002-8361-9815

Yoon Hee Kim http://orcid.org/0000-0002-2149-8501

Kyung Won Kim http://orcid.org/0000-0003-4529-6135

Myung Hyun Sohn http://orcid.org/0000-0002-2478-487X

Kyu-Earn Kim http://orcid.org/0000-0002-5730-3331

\section{References}

1) Hwang HS, Lee NY, Han SB, Kwak GY, Lee SY, Chung SY, et al: Performance effectiveness of pediatric index of mortality 2 (PIM2) and pediatricrisk of mortality III (PRISM III) in pediatric patients with intensive care in single institution: Retrospective study. Korean J Pediatr 2008; 51: 1158-64.

2) Leteurtre S, Martinot A, Duhamel A, Proulx F, Grandbastien B, Cotting J, et al: Validation of the paediatric logistic organ dysfunction (PELOD) score: prospective, observational, multicentre study. Lancet 2003; 362: 192-7.

3) Taori RN, Lahiri KR, Tullu MS: Performance of PRISM (Pediatric Risk of Mortality) score and PIM (Pediatric Index of Mortality) score in a tertiary care pediatric ICU. Indian J Pediatr 2010; 77: 267-71.

4) Sankar J, Chandel A, Dubey NK, Sreenivas V, Sankar MJ: Do interventions in an ICU affect the predictive ability of pediatric index of mortality and pediatric index of mortality- 2 scores in a tertiary care hospital? Pediatr Crit Care Med 2013; 14: e70-6.

5) Wolfler A, Osello R, Gualino J, Calderini E, Vigna G, Santuz P, et al: The importance of mortality risk assessment: Validation of the pediatric index of mortal- 
ity 3 score. Pediatr Crit Care Med 2016; 17: 251-6.

6) Lobo SM, Lobo FR, Bota DP, Lopes-Ferreira F, Soliman HM, Mélot C, et al: C-reactive protein levels correlate with mortality and organ failure in critically ill patients. Chest 2003; 123: 2043-9.

7) Vanderschueren S, De Weerdt A, Malbrain M, Vankersschaever D, Frans E, Wilmer A, et al: Thrombocytopenia and prognosis in intensive care. Crit Care Med 2000; 28: 1871-6.

8) Kim YH, Park HB, Kim MJ, Kim HS, Lee HS, Han YK, et al: Usefulness of thrombocytopenia and changes in platelet counts as prognostic markers in pediatric intensive care units. Korean J Crit Care Med 2013; 28: 93-100.

9) Jensen JU, Heslet L, Jensen TH, Espersen K, Steffensen P, Tvede M: Procalcitonin increase in early identification of critically ill patients at high risk of mortality. Crit Care Med 2006; 34: 2596-602.

10) Devakonda A, George L, Raoof S, Esan A, Saleh A, Bernstein LH: Transthyretin as a marker to predict outcome in critically ill patients. Clin Biochem 2008; 41: 1126-30.

11) Abidi K, Belayachi J, Derras Y, Khayari ME, Dendane T, Madani N, et al: Eosinopenia, an early marker of increased mortality in critically ill medical patients. Intensive Care Med 2011; 37: 1136-42.

12) Nahm CH, Choi JW, Lee J: Delta neutrophil index in automated immature granulocyte counts for assessing disease severity of patients with sepsis. Ann Clin Lab Sci 2008; 38: 241-6.

13) Harris N, Kunicka J, Kratz A: The ADVIA 2120 hematology system: flow cytometry-based analysis of blood and body fluids in the routine hematology laboratory. Lab Hematol 2005; 11: 47-61.

14) Kratz A, Maloum K, O’Malley C, Zini G, Rocco V, Zelmanovic D, et al: Enumeration of nucleated red blood cells with the ADVIA 2120 Hematology System: an International Multicenter Clinical Trial. Lab
Hematol 2006; 12: 63-70.

15) Park BH, Kang YA, Park MS, Jung WJ, Lee SH, Lee SK, et al: Delta neutrophil index as an early marker of disease severity in critically ill patients with sepsis. BMC Infect Dis 2011; 11: 299.

16) Park HJ, Ha YJ, Pyo JY, Park YB, Lee SK, Lee SW: Delta neutrophil index as an early marker for differential diagnosis of adult-onset Still's disease and sepsis. Yonsei Med J 2014; 55: 753-9.

17) Ansari-Lari MA, Kickler TS, Borowitz MJ: Immature granulocyte measurement using the Sysmex XE2100. Relationship to infection and sepsis. Am J Clin Pathol 2003; 120: 795-9.

18) Bernstein LH, Rucinski J: Measurement of granulocyte maturation may improve the early diagnosis of the septic state. Clin Chem Lab Med 2011; 49: 208995.

19) Nigro KG, O’Riordan M, Molloy EJ, Walsh MC, Sandhaus LM: Performance of an automated immature granulocyte count as a predictor of neonatal sepsis. Am J Clin Pathol 2005; 123: 618-24.

20) Pollack MM, Patel KM, Ruttimann UE: PRISM III: an updated Pediatric Risk of Mortality score. Crit Care Med 1996; 24: 743-52.

21) Slater A, Shann F, Pearson G: PIM2: a revised version of the Paediatric Index of Mortality. Intensive Care Med 2003; 29: 278-85.

22) Straney L, Clements A, Parslow RC, Pearson G, Shann F, Alexander J, et al: Paediatric index of mortality 3: an updated model for predicting mortality in pediatric intensive care*. Pediatr Crit Care Med 2013; 14: 673-81.

23) Hanley JA, McNeil BJ: The meaning and use of the area under a receiver operating characteristic (ROC) curve. Radiology 1982; 143: 29-36.

24) Youden WJ: Index for rating diagnostic tests. Cancer 1950; 3: 32-5. 